\title{
Research Project of TEAMS for Restoration of Ecosystems and Human Society
}

\author{
Akihiro Kijima \\ Representative of TEAMS (Tohoku Ecosystem-Associated Marine Science) Japan
}

Keywords: 2011 Great East Japan Earthquake and Tsunami, restoration, ecosystems, human society, TEAMS

The Great East Japan Earthquake and Tsunami on March 11, 2011, caused immense damage to marine ecosystems, both nearshore and offshore, on the Pacific coast of northeastern Japan (the Tohoku region). Most fishing vessels and fishery related infrastructure were destroyed by the disaster. In the Tohoku region, salmon hatcheries have been common in many rivers, and every year, released chum salmon have returned to their own rivers. When the 2011 disaster occurred, a lot of juvenile chum salmon were reared in the hatcheries before being released into the sea. Therefore, the salmon production cycle in this region was also severely disrupted by the disaster.

In order to reconstruct the devastated human society, scientific investigation was essential to understand the effects of such strong disturbances on not only coastal land areas but marine ecosystems, and to monitor the process of their recovery. The research project of "Tohoku Ecosystem-Associated Marine Sciences (TEAMS)" was established under such circumstances by the Ministry of Education, Culture, Sports, Science and Technology of Japan (MEXT) in 2011FY. The purpose of the TEAMS project is to clarify the impact of the earthquake and subsequent tsunami on marine-related ecosystems of the Tohoku coastal areas, highlight the restoration process of the ecosystems based on scientific research, and contribute to the reconstruction of fishery industries in the Tohoku region, including salmon production.

The TEAMS project was carried out for a period of 10 years - ranging from coastal to offshore seas and from surface to bottom in the Sanriku region-involving the cooperation and collaboration of a large number of researchers across Japan. Enormous research results obtained on the catastrophe of the earthquake and tsunami on the land and ocean ecosystems will contribute to future crisis management know-how not only in Japan but also overseas (TEAMS 2016). The background and results of this project will be a role model for disaster recovery as it included collaboration between different fields and regions and cooperation between researchers, fishermen, research institutes, and governments both local and national.

In addition, the results of this project will be useful for the monitoring and future prediction of marine ecosystem dynamics, the development of sustainable fisheries and aquaculture management, and the establishment of a new marine industry coexisting with marine environmental conservation and landscape (TEAMS 2020). We hope that they will be widely useful in various fields such as the education for conservation of marine ecosystems.

\section{REFERENCES}

Tohoku Ecosystem-Associated Marine Sciences (TEAMS). 2016. Marine ecosystems after Great East Japan Earthquake in 2011. Tokai University Press, Tokyo. 162 pp.

Tohoku Ecosystem-Associated Marine Sciences (TEAMS). 2020. Achievement report of Tohoku EcosystemAssociated Marine Sciences: research on marine ecosystem. 91 pp. (In Japanese) 